\title{
OH AS A CONSTITUENT OF THE INTERSTELLAR MEDIUM
}

\author{
B. E. TURNER \\ National Radio Astronomy Observatory*, Green Bank, West Virginia, U.S.A.
}

\begin{abstract}
OH}$ is at present the most ubiquitous molecule known in the interstellar medium. It is primarily associated with galactic continuum sources, both thermal and nonthermal. The fraction of all sources showing $\mathrm{OH}$ decreases monotonically with galactic longitude away from the Galactic Center region. However, neither the projected density of $\mathrm{OH}$ nor the abundance relative to hydrogen or other molecules seems to depend on location within the galactic plane. $\mathrm{OH}$ also occurs in many dark dust clouds, where its density is typically $10^{-5}-10^{-4} \mathrm{~cm}^{-3}$ and its abundance relative to hydrogen is very high. $\mathrm{OH}$ is not found in the heavily reddened stellar clusters of Reddish, in Wolf Rayet stars, in planetary nebulae, or in globules. Recent surveys have indicated that $\mathrm{OH}$ both in emission and in absorption, is highly correlated with $\mathrm{H}_{2} \mathrm{CO}$ in direction and in velocity. $\mathrm{OH}$ emission, but not absorption, is also highly correlated with anomalous $\mathrm{H}_{2} \mathrm{O}$ emission. These relationships are important in deciding between various processes for interstellar molecule formation. On present infor. mation, it appears that more than one such process is operative in different regions of the interstellar medium.
\end{abstract}

\section{Introduction}

At present, the $\mathrm{OH}$ molecule has been detected in absorption or emission, or both, in more than 100 galactic regions. The $\mathrm{OH}$ seen in absorption appears to be associated generally with $\mathrm{HI}$ regions and hence originates in the spiral arms delineated by the neutral hydrogen. The $\mathrm{OH}$ seen in both absorption and emission also generally agrees with the spiral arms as delineated by Hur regions.

In this review, we shall not primarily be concerned with the galactic structure aspect of $\mathrm{OH}$. Instead we consider the problems of its abundance, its relation to other molecules and to hydrogen, its physical association with other types of objects, and the question of formation and destruction. The problem of the anomalous excitation of $\mathrm{OH}$ will also not be discussed except where it may be relevant to the question of formation.

\section{Distribution of $\mathrm{OH}$ in the Galaxy; Physical Associations}

As is well-known, the $\mathrm{OH}$ is concentrated toward the galactic plane, as the measured optical depths for the absorption sources decrease significantly with increasing galactic latitude (Goss, 1968). In this respect there is a selection effect; many more low-latitude sources have been investigated than high-latitude ones. However, the largest mean optical depth known so far is that in Ori $\mathrm{B}$, at a latitude of $-16^{\circ}$.

The $\mathrm{OH}$ distribution in the plane shows a marked decrease with longitude in the interval $345^{\circ} \leqslant l \leqslant 133^{\circ}$ covered by northern surveys. For this trend to be clear, one must refer to the recent survey by Turner (1970) which covered many relatively weak continuum sources whereas all previous surveys have been strongly selective toward only the strongest continuum sources. The southern hemisphere surveys of Robinson

* Operated by Associated Universities, Inc., under contract with the National Science Foundation, 
et al. (1970); Goss et al. (1970) and Manchester et al. (1970) are also limited in this respect. When one compiles all existing surveys of the northern hemisphere, the most obvious correlation that emerges is that the very strongest HII regions are more likely to have $\mathrm{OH}$ associated with them than are the weaker ones; or at least that the strongest $\mathrm{OH}$ emission or absorption lines, hence the $\mathrm{OH}$ most likely to be detected with the earlier less sensitive equipment, is associated with the strongest continuum sources.

However, if one combines the surveys of Goss (1968) and Turner (1970), both of which concentrated only on continuum sources (nonthermal or thermal), one finds the following results.

(1) The fraction of continuum sources associated with $\mathrm{OH}$ emission or absorption decreases monotonically as $l$ increases from $345^{\circ}$ through $0^{\circ}$ to $75^{\circ}$. The fraction, with corresponding $l$ range, is as follows: $\frac{16}{18}(345 \leqslant l \leqslant 355), \frac{5}{6}(355 \leqslant l \leqslant 5)$, $\frac{1}{15}(5 \leqslant l \leqslant 15), \frac{9}{15}(15 \leqslant l \leqslant 25), \frac{4}{8}(25 \leqslant l \leqslant 35), \frac{2}{5}(35 \leqslant l \leqslant 45), \frac{2}{11}(45 \leqslant l \leqslant 75)$. In these we have included some previous negative results (Turner, 1969b).

(2) The distribution of emission sources alone does not follow the above rule, but in fact seems quite random except for a notable group of five which falls in the range $18.8 \leqslant l \leqslant 20.0$. This group is adjacent to the interval $15.2 \leqslant l \leqslant 18.5$ which contains no $\mathrm{OH}$ sources of any type, out of five searched. Neither of these ranges of $l$ includes tangent points for spiral arms.

(3) That the $\mathrm{OH}$ is generally physically associated with the continuum sources is shown by the high correlation of $\mathrm{OH}$ velocities and recombination line velocities. There is agreement to within $5 \mathrm{~km} / \mathrm{sec}$ in 10 out of 16 absorption line sources with $l>5^{\circ}, 4$ out of 12 absorption line sources with $l<5^{\circ}$, and 6 out of 14 emission line sources overall. This correlation means that the $\mathrm{OH}$ velocities may also be expected to follow roughly the galactic rotation curve, and this is found to be the case when we include also the more than $50 \%$ of $\mathrm{OH}$ sources seen against continuum sources which have not as yet displayed recombination lines.

On the other hand, for $l$ within $5^{\circ}$ of the Galactic Center it is found that the $\mathrm{OH}$ velocities are distributed roughly uniformly over the range -20 to $+20 \mathrm{~km} / \mathrm{sec}$. It is not known whether the scatter here is larger than it is for larger $l$, and hence whether the quite good agreement of most $\mathrm{OH}$ velocities at larger $l$ with the rotation curve is somewhat fortuitous.

(4) The absorption linewidths are not correlated with either $T_{c}$ for the continuum sources or with position in the Galaxy, although there is an indication that the rms scatter in linewidths, for absorption sources, may be less for $l<10^{\circ}$ than for $l>10^{\circ}$. Somewhat over half of all absorption lines appear to be single features, so that lack of correlation with $T_{c}$ may suggest the $\mathrm{OH}$ lies outside the ionized region, as would be expected also on grounds of survival of the $\mathrm{OH}$.

\section{Physical Associations}

In addition to being associated with $\mathrm{H}$ II regions, there is probably a diffuse com- 
ponent of $\mathrm{OH}$ distributed throughout the spiral arms. This may be gathered from the observation of the local $\mathrm{OH}$ gas in our own spiral arm, which has been observed in absorption against a few strong extragalactic or nonthermal galactic sources (Cyg A, $3 \mathrm{C} 123$, Tau A, Cas A). These observations show a column density of $\mathrm{OH}$ which is considerably smaller than is observed in the direction of most HiI regions, but an $\mathrm{OH} / \mathrm{H}$ ratio which is probably only slightly smaller. No extensive attempts have been made to detect a general $\mathrm{OH}$ component away from continuum sources in other spiral arms.

As has been indicated, the strongest concentrations of $\mathrm{OH}$ clearly tend to be associated with continuum sources, especially thermal ones. Altogether, some $80 \%$ of thermal sources included in the surveys of Goss (1968) and Turner (1970) have OH. The corresponding number for non-thermal sources is $69 \%$. While it is obvious that one sees absorption lines only if continuum sources are present, nonetheless the velocity correspondence between $\mathrm{OH}$ and recombination lines shows the relation to be a true one, at least for thermal sources. Regarding $\mathrm{OH}$ emission sources, there has again been a strong selection effect toward observing in the direction of continuum sources. However, at least one survey (Turner, 1969b) has avoided continuum sources with negative results. That survey covered several different classes of objects which might have been expected to show $\mathrm{OH}$. These objects included.

(a) - the heavily reddened stellar clusters and associations of Reddish (1967). These are thought to be extremely young, contain an abundance of dust and probably grains, and involve young hot stars whose UV flux might be suitable for anomalous excitation of $\mathrm{OH}$.

(b) - Wolf-Rayet stars with and without surrounding nebulosity. If these stars are indeed very young, they might have been appropriate sites for the formation of $\mathrm{OH}$, which is itself usually considered to be associated only with the youngest $\mathrm{H}$ II regions.

(c) - Hil regions from Sharpless' list (1959) which are not apparent radio sources. The lack of $\mathrm{OH}$ in these sources may merely be because they are relatively old $\mathrm{HI}$ regions.

(d) - globules. It is somewhat puzzling why no molecules have ever been observed from these presumably dense, cool objects. The upper limit for the strength of $\mathrm{OH}$ lines is small enough to have allowed detection of $\mathrm{OH}$ if its strength were typical of that of most dust clouds. From a molecular viewpoint, it is therefore not clear that the globules are an evolutionary link with dust clouds.

(e) - planetary nebulae. Lack of $\mathrm{OH}$ in the direction of these objects shows that the existence of a radio continuum source is not sufficient for the presence of $\mathrm{OH}$. It further argues that $\mathrm{OH}$ is associated only with young objects.

For the majority of the above objects which lie nowhere near continuum sources, the negative result of this survey may be taken to show not only that these types of objects are not themselves regions of enhanced molecular activity, but also that there is no weak, generally extended disk of $\mathrm{OH}$ which might have been detected away from continuum sources. In addition, the continuum sources associated with three of the 
Wolf-Rayet stars and with the planetary nebulae would have allowed detection of quite weak $\mathrm{OH}$ in absorption if any had been present.

Since the strongest concentrations of $\mathrm{OH}$ seem to be associated mainly with $\mathrm{H}_{\text {II }}$ regions, we might inquire whether there is anything in the detailed structure of these sources which distinguishes them from those few $\mathrm{H}$ II regions without $\mathrm{OH}$ or from planetary nebulae which are not associated with $\mathrm{OH}$.

To answer this question we summarize some results obtained at $11 \mathrm{~cm}$ wavelength by Webster and Altenhoff (1970) and by Turner et al. (1970) using the 3-element interferometer of the NRAO. These results show first that virtually all $\mathrm{HII}$ regions, with and without $\mathrm{OH}$, contain considerable fine structure in the continuum, on the order of $20^{\prime \prime}$ and smaller in size. A similar statement may be made for NGC 7027, the only planetary nebula thus far synthesized. Only the $\mathrm{OH}$ sources in anomalous emission have accurate enough positions (determined by VLB measurements) to compare with the detailed continuum structure of the Hil regions. In one case (W49) both $\mathrm{OH}$ emission regions appear to coincide in projection with small continuum knots. In W51 a very small continuum knot ( $\lesssim 0^{\prime \prime} .6$ in size) found by Miley et al. (1970) is 8" from the $\mathrm{OH}$ emission center and is probably physically associated. The $\mathrm{W} 3 \mathrm{OH}$ emission source apparently coincides with a very small and weak continuum source. However, in Ori A there is no correspondence of $\mathrm{OH}$ emission centers with fine continuum features, although one of the $\mathrm{OH}$ emission centers coincides with the Becklin and Neugebauer IR star within the positional uncertainties of the various objects.

For the large majority of $\mathrm{HII}$ regions which have been sampled but not synthesized by interferometry, the visibility characteristics may be compared statistically with the incidence of $\mathrm{OH}$. Turner et al. (1970) have examined the continuum of 11 of the new sources found to have $\mathrm{OH}$ emission, 11 having $\mathrm{OH}$ absorption, and 3 having no $\mathrm{OH}$. Lobe spacings were 113,12 , and $11 \mathrm{arcsec}$, at a wavelength of $11 \mathrm{~cm}$. One quantity of significance is the ratio, $f$, of flux as measured at the 11 arc sec spacing and at $113 \mathrm{arcsec}$ spacing. There is no statistical difference in $f$ between the sources with and without $\mathrm{OH}$, or with $\mathrm{OH}$ emission as distinct from absorption. Another significant parameter is the total flux contained in structure of size $113 \mathrm{arcsec}$ and less, since this is a good measure for considering whether a source is 'compact' or not. Again there is no distinction between the sources with $\mathrm{OH}$ emission, $\mathrm{OH}$ absorption, or no $\mathrm{OH}$. There would appear to be no distinction between the continuum sources with $\mathrm{OH}$ emission and absorption; the previously proposed association of $\mathrm{OH}$ emission sources and compact $\mathrm{HII}$ regions merely reflects the more detailed study which these sources had received.

\section{The Abundance of $\mathrm{OH}$}

It is the case with all interstellar molecules observed only at radio wavelengths that abundances are difficult to determine with any reliability. The reasons are lack of knowledge of (a) the fraction $R$ of the background continuum source covered by the absorbing cloud and (b) the excitation temperature $T_{s}$, or even whether there is a 
unique value of $T_{s}$ for all transitions. The latter problem arises because of the ease with which the populations in energy levels with $h v \ll k T$ can be significantly perturbed from the LTE populations when collision rates do not dominate radiative rates.

In the case of $\mathrm{OH}$, it is well-known that abundance determinations are impossible for the anomalous emission sources, for which we do not even know whether the maser amplification is saturated or unsaturated. It has more recently become clear that the absorption sources also display significant departures from LTE and therefore also cannot yield reliable abundances.

In determining abundances, the only directly measured quantity is $\langle\tau\rangle$, the apparent optical depth, which is related to the true optical depth $\tau$ by

$$
1-\exp (-\langle\tau\rangle(v))=R[1-\exp (-\tau(v))]
$$

Thus $\tau$ is derivable only if $R$ is known. Then

$$
\frac{N_{\mathrm{OH}}}{T_{s}}=\frac{8 \pi k v}{h c^{2} A} \frac{\sum_{i} g_{i}}{g_{u}} \int_{-\infty}^{\infty} \tau(v) \mathrm{d} v .
$$

If $\langle\tau\rangle$ is used rather than $\tau$ the resultant $N_{\mathrm{OH}} / T_{s}$ is averaged over the continuum source. When only a single absorption line is available, $N_{\mathrm{OH}}$ and $T_{s}$ cannot be derived separately. Note that this expression assumes $h v / k(0.08 \mathrm{~K}) \ll T_{s}$.

When two absorption lines are used, the problem of the value of $R$ is avoided, since the ratio of measured antenna temperatures is simply

$$
\frac{\Delta T_{A 1}(v)}{\Delta T_{A 2}(v)}=\frac{1-\exp \left(-\langle\tau\rangle_{1}(v)\right)}{1-\exp \left(-\langle\tau\rangle_{2}(v)\right)}=\begin{aligned}
& 1-\exp \left(-\tau_{1}(v)\right) \\
& 1-\exp \left(-\tau_{2}(v)\right)
\end{aligned}
$$

where $\tau_{1}(v)$ and $\tau_{2}(v)$ are related by the ratio of line strengths, assuming $T_{s}$ is the same for both lines. In addition, $T_{s}$ could in principle be determined next from the relation $\Delta T_{A 1}=\eta_{B}\left(T_{s}-T_{B c}\right)\left[1-\exp \left(-\left\langle\tau_{1}\right\rangle(v)\right)\right]$ provided $\eta_{B}$ and $T_{B c}$, the background continuum brightness temperature, could be found with sufficient accuracy. Generally this is not the case.

A third way by which $N_{\mathrm{OH}}$ may be derived for a single transition, if the ratios suggest non-LTE excitation, is to derive $T_{s}$ for the line in question by observing it in emission at a position off the continuum source. This method requires knowledge of $R$ however, and also assumes $T_{s}$ does not differ in the absorbing and emission parts of the cloud. Ori B is a good case for this method. Since the background source is quite large and the lines are strong, mapping the absorption allows a good determination of $R$. Also, Davies (1969) and Manchester and Gordon (1970) have found weak emission at $1667 \mathrm{MHz}$ well off the continuum source, which should allow a good estimate of $T_{s}$. 
In general, owing to the above-mentioned difficulties, it has been customary to derive an estimate of $N_{\mathrm{OH}} / T_{s}$ by using (I) except that $\langle\tau\rangle(v)$ is used in the integral rather than $\tau(v)$. Thus the estimate is a strong lower limit to $N_{\mathrm{OH}} / T_{s}$. It has then usually been assumed that $T_{s} \approx 10 \mathrm{~K}$ to derive $N_{\mathrm{OH}}$.

That this method seriously underestimates $N_{\mathrm{OH}} / T_{s}$ may be seen for the case of Ori B. This source shows the largest measured optical depth $\langle\tau\rangle$ (by about a factor of 4) of any source so far observed in absorption. $\langle\tau\rangle$ at $1667 \mathrm{MHz}$ is found to be $\sim 0.44$ (Goss, 1968), while the ratio of 1667 to $1665 \mathrm{MHz}$ lines implies that the true optical depth in the I667 line, $\tau(1667)$, is $>20$. Differences in $T_{s}$ for the 4 lines are indisputable for this source, since the ratio $1667 / 1665$ implies a value of $\tau(1667)$ which is inconsistent with the value derived from the ratio $1667 / 1612$. However if $\tau(1667)$ is indeed large, as seems very likely, then $R \approx 1-e^{-\langle\tau\rangle}=0.35$; the $\mathrm{OH}$ cloud covers $\sim 35 \%$ of the source. Since the source is $4^{\prime}$ to half-power according to Mezger and Henderson (1967), the characteristic size of the $\mathrm{OH}$ cloud is $\sim 1$ '.5. This result is consistent with Clark's (1965) conclusion regarding $\mathrm{HI}$ absorption in Ori A; he suggests that the absorption is due to a $4^{\prime} \mathrm{HI}$ feature of $\tau>2$.

In spite of the above difficulties, use of (1) (with $\langle\tau\rangle$ instead of $\tau$ ) is all that has been done in the majority of sources whose line ratios in absorption are close enough to LTE to make an estimate of $N_{\mathrm{OH}} / T_{s}$ profitable. We now summarize the results.

\section{A. OH ASSOCIATED WITH CONTINUUM SOURCES}

Goss (1968) observed absorption in 37 sources in the northern hemisphere. Of these, $N_{\mathrm{OH}} / T_{s}$ (in units of $10^{13} \mathrm{~cm}^{-2} \mathrm{~K}^{-1}$ has a value $<1$ for 3 sources, 1 to 3 for 11 sources, 3 to 5 for 6 sources, 5 to 7 for 5 sources, 7 to 9 for 4 sources, 9 to 11 for 3 sources, and $>11$ for 5 sources. The value of $N_{\mathrm{OH}} / T_{s}$ is not correlated with distance from the Sun, nor with distance from the galactic center. Since an $85 \mathrm{ft}$ telescope with beamwidth $\sim 33^{\prime}$ was used, these sources were badly confused by the galactic background, and the opacities therefore are underestimated by a greater factor than if a smaller beamwidth were employed. In this regard, a comparison of the above survey with that of Robinson et al. (1970) who used a $12^{\prime}$ beamwidth, is interesting. There are only 4 sources in common, comprising 8 different features. There seems to be a tendency for weak absorption features (small values of $N_{\mathrm{OH}} / T_{s}$ ) to yield the same value of $N_{\mathrm{OH}} / T_{\mathrm{s}}$ in both surveys, while the stronger absorption features give larger values of $N_{\mathrm{OH}} / T_{s}$ when observed with the $12^{\prime}$ beam (by typically a factor of 2 , but less than the ratio of beam areas). Interpreted literally, this suggests the weak features are quite extended, while the strong ones are less so, presumably being smaller than the $12^{\prime}$ beam used by Robinson et al. However, the apparent smaller size of the stronger absorption clouds might as well result from the (accidental) characteristics of the background sources as seen by the two different beams, as to the absorbing $\mathrm{OH}$ clouds themselves. There is strong need for a systematic survey of more absorption sources with two or more telescopes of significant difference in size to resolve this question. It might be noted that at least one $\mathrm{OH}$ absorption feature, in W43, seems to be very extended (Turner, 1969a) and that two other sources, W28 and W44, show 
$\mathrm{OH}$ absorption with the same velocity at widely separated points $\left(\sim 18^{\prime}\right)$ and presumably in between as well, although a map of the absorption with a sufficiently fine grid spacing has not been done.

The surveys of Goss et al. (1970) and Manchester et al. (1970) of southern hemisphere sources establish the following results for $N_{\mathrm{OH}} / T_{s}\left(\times 10^{13} \mathrm{~cm}^{-2} \mathrm{~K}^{-1}\right)$ : a value 1-3 for 3 sources, 7-9 for 1 source, $9-11$ for 1 source, and $>11$ for 7 sources. Again there is no dependence of $N_{\mathrm{OH}} / T_{s}$ on position in the Galaxy.

The recent survey by Turner (1970) of the discrete continuum sources found at $11 \mathrm{~cm}$ in the survey of Altenhoff et al. (1970) has uncovered 50 new $\mathrm{OH}$ sources of which 28 show only absorption of the $\mathrm{OH}$. The range of values of $N_{\mathrm{OH}} / T_{s}$ is the same as for the previous sources. If we consider these and all previously known $\mathrm{OH}$ absorption sources together, we draw the following conclusions about the abundance distribution of $\mathrm{OH}$ in the Galaxy:

(1) There is no correlation between apparent opacity $\langle\tau\rangle$ and continuum brightness temperature $T_{c}$.

(2) There is no correlation between $\langle\tau\rangle$ and position in the Galaxy.

Regarding the anomalous $\mathrm{OH}$ emission sources, every indication is that the projected $\mathrm{OH}$ densities are considerably higher than in the sources showing $\mathrm{OH}$ only in absorption. Direct observational evidence exists for this conclusion in the case of Class II(a) emission sources for which the two main lines evidently have normal populations and only the $1720 \mathrm{MHz}$ line is in emission, at the same velocity. Two examples of this type of source are W28 and W44. In these sources, the value of $\langle\tau\rangle$ derived from the absorption is weaker by a factor of 2.6 and 1.6 respectively at the continuum maximum, where there is no $1720 \mathrm{MHz}$ emission, than at points well off the maximum where the $1720 \mathrm{MHz}$ emission occurs (Turner, 1969a). For Class I and II(b) emission sources, which do not have $\mathrm{OH}$ absorption at the same velocity as the emission, no observational evidence on this question is possible. Theoretically, it has proved difficult to account for the observed brightness temperatures unless the column densities in the emission regions are at least $10^{2}-10^{3}$ higher than are the (lower limit) estimates for the absorption sources. For example, the far-IR pumping mechanisms that appear to explain the type II(a) emission sources (Litvak, 1969) depend only on the quantity $N_{\mathrm{OH}} v / \delta v$ ( $\delta v$ is the linewidth) and lead to values for $N_{\mathrm{OH}}$ of $10^{16}-10^{17}$ when the kinetic temperature is $\sim 100 \mathrm{~K}$. (Of course, the IR rates must dominate collision rates, which means that total particle densities must not exceed $\sim 3 \times 10^{5}$ $\mathrm{cm}^{-3}$; if the maser path length is that of the VLB-measured linear size of the emitting region, $\mathrm{OH}$ densities would be typically $10^{2}-10^{3} \mathrm{~cm}^{-3}$, so that the abundance of $\mathrm{OH}$ relative to other constituents in these regions would be at least $10^{-3}$ and probably much higher.)

There is very little information bearing on the question of $\mathrm{OH} / \mathrm{H}$ ratios. Assuming $T_{s}(\mathrm{OH})=10 \mathrm{~K}$, Weinreb et al. (1963) find $\mathrm{OH} / \mathrm{H}=10^{-7}$ for the absorbing clouds in front of Cas A, and Robinson et al. (1964) find $\mathrm{OH} / \mathrm{H}$ from $8 \times 10^{-7}$ to $>10^{-4}$ for three different absorption features in the $\mathrm{Sgr} \mathrm{A}$ region. For the few $\mathrm{HII}$ regions in the directions of which there is high resolution $21-\mathrm{cm}$ absorption line data, we find 
$\mathrm{OH} / \mathrm{H}=1.1 \times 10^{-5}$ (Ori B), $0.9 \times 10^{-6}(\mathrm{M} 17),<1.0 \times 10^{-6}(\mathrm{~W} 22),<2.8 \times 10^{-7}(\mathrm{~W} 29)$, $<1.2 \times 10^{-6}$ (W37), $5.2 \times 10^{-7}$ (W43), and $0.9 \times 10^{-6}(\mathrm{~W} 51)$.

\section{B. OH IN DUST CLOUDS}

Until recently the weak OH emission observed by Heiles $(1968,1969)$ in dust clouds was thought to be thermal, since the only lines then observed, the main lines, were in the LTE ratio 9:5 or less depending on the optical depth. Recently Turner and Heiles (1970) have found that the satellite lines are not in LTE, but exhibit a weak version of the anomaly characterizing type II(a) emission sources - an enhanced emission at $1720 \mathrm{MHz}$ and suppressed emission at $1612 \mathrm{MHz}$. These anomalies do not invalidate the use of the main lines deriving $N_{\mathrm{OH}}$ and $T_{s}$ however. Furthermore, because the dust clouds are typically larger than the beamwidth used in the observations and the line ratios are found to be constant at all positions measured in these clouds, the line ratio technique described above leads to an unambiguous determination of $N_{\mathrm{OH}}$ and $T_{s} . N_{\mathrm{OH}}$ is found to be $2.4 \times 10^{15} \mathrm{~cm}^{-2}$ for Cloud 2 and $7.2 \times 10^{14}$ $\mathrm{cm}^{-2}$ for Cloud $4 \mathrm{C}$ while $T_{s}$ is $3.65 \mathrm{~K}$ and $5^{\circ} .44 \mathrm{~K}$ respectively. If the cloud dimensions along the line of sight are the same as the transverse dimensions, the $\mathrm{OH}$ density is $5.1 \times 10^{-5} \mathrm{~cm}^{-3}$ and $2.2 \times 10^{-4} \mathrm{~cm}^{-3}$ respectively.

\section{The Association of $\mathrm{OH}$ with Other Interstellar Molecules}

Sufficient survey work has now been done to indicate the degree of correlation of $\mathrm{OH}$ with $\mathrm{H}_{2} \mathrm{O}$ and $\mathrm{H}_{2} \mathrm{CO}$ throughout the galactic plane, although data on $\mathrm{CO}, \mathrm{CN}$, $\mathrm{NH}_{3}$, and $\mathrm{HCN}$ are preliminary at present.

The earliest trend noted was the complete correlation of $\mathrm{H}_{2} \mathrm{O}$ sources with $\mathrm{OH}$ emission sources of type $\mathrm{I}$ in $\mathrm{H}$ II regions. More recently, a few weak $\mathrm{H}_{2} \mathrm{O}$ emission sources have been found in IR stars which have no $\mathrm{OH}$ counterparts, but even in the IR star sources, $\mathrm{OH}$ emission usually accompanies $\mathrm{H}_{2} \mathrm{O}$ emission. It is significant that no $\mathrm{H}_{2} \mathrm{O}$ sources have been found coincident with $\mathrm{OH}$ absorption sources, despite searches in several dozen sources. The fact that $\mathrm{H}_{2} \mathrm{O}$ so commonly accompanies $\mathrm{OH}$ when both are in emission suggests that it may also exist in $\mathrm{OH}$ absorption clouds but be undetectable owing to inadequate excitation of the $6_{16}$ rotational level, which requires $477 \mathrm{~cm}^{-1}$ energy. In the $\mathrm{OH} / \mathrm{H}_{2} \mathrm{O}$ emission sources, it is thought that the $\mathrm{H}_{2} \mathrm{O}$ abundance is a good deal larger than the $\mathrm{OH}$ abundance, owing to the much larger power radiated by the $\mathrm{H}_{2} \mathrm{O}$ masers.

$\mathrm{OH}$ is also highly correlated with $\mathrm{H}_{2} \mathrm{CO}$ over the galactic plane. In comparing the $\mathrm{H}_{2} \mathrm{CO}$ survey of Zuckerman et al. (1970) with the $\mathrm{OH}$ survey of Goss (1968), we find a total of 6 sources showing $\mathrm{OH}$ but no $\mathrm{H}_{2} \mathrm{CO}$, and no sources showing $\mathrm{H}_{2} \mathrm{CO}$ but no $\mathrm{OH}$, out of 41 sources in common. A similar comparison of the 39 sources common to the $\mathrm{OH}$ survey of Turner (1970) and to the recent unpublished $\mathrm{H}_{2} \mathrm{CO}$ survey by Wilson (1970) shows that 28 sources have both $\mathrm{OH}$ and $\mathrm{H}_{2} \mathrm{CO}, 5$ have only $\mathrm{OH}$, 2 have only $\mathrm{H}_{2} \mathrm{CO}$, and 4 have neither. From these surveys we see that $\mathrm{OH}$ is perhaps slightly more widespread than $\mathrm{H}_{2} \mathrm{CO}$. Wilson (1970) also finds evidence for $\mathrm{H}_{2} \mathrm{CO}$ 
away from several of the continuum sources, with a highly patchy distribution. It is entirely probable that $\mathrm{OH}$ also exists away from the continuum sources but has not been detected there as yet, since it lacks the anomalous absorption qualities of $\mathrm{H}_{2} \mathrm{CO}$.

Data for the more recently discovered molecules is very incomplete. $\mathrm{CO}$ has been found in 5 sources, all of which show $\mathrm{OH}$, but the selection toward $\mathrm{OH}$ sources in the search (Wilson et al., 1970) was complete. Nevertheless it is interesting that the $\mathrm{CO}$ emission in the Ori A region extends far off the continuum source, and must arise in the $\mathrm{Hi}$ region. Very weak $\mathrm{OH}$ emission, of a presumably thermal character (as is probably the $\mathrm{CO}$ emission), was found well off the Ori A continuum source by Davies (1969). The $5 \mathrm{CN}$ and $6 \mathrm{HCN}$ sources presently known are also $\mathrm{OH}$ emission sources.

In the dust clouds, $\mathrm{H}_{2} \mathrm{CO}$ again correlates highly with $\mathrm{OH}$ although often at a velocity differing by a few $\mathrm{km} \mathrm{sec}^{-1}$. Here, $\mathrm{H}_{2} \mathrm{CO}$ is found in a few clouds which do not show $\mathrm{OH} . \mathrm{H}_{2} \mathrm{O}, \mathrm{CO}$, and $\mathrm{CN}$ are as yet undetected in dust clouds. $\mathrm{HCN}$ occurs in Cloud 2, which also shows $\mathrm{OH}$.

The relative abundances of the various molecules is very poorly known. If we assume $T_{s}$ for $\mathrm{H}_{2} \mathrm{CO}$ is $\approx 3 \mathrm{~K}$ for all $\mathrm{H}$ II regions, then the survey of Zuckerman et al. yields an $\mathrm{OH} / \mathrm{H}_{2} \mathrm{CO}$ ratio which varies between $\approx 10$ and 100 over a total of 15 sources for which a comparison is possible. The column density of $\mathrm{CO}$ in Ori A must be 1000 times greater than that of $\mathrm{OH}$, but the ratio of actual densities is probably much smaller, perhaps of order unity. (The column densities in the five known $\mathrm{CO}$ sources appear to be anti-correlated with the $\mathrm{H}_{2} \mathrm{CO}$ column densities.)

It appears that any mechanism for the formation of $\mathrm{OH}$ must also be able to account for the $\mathrm{H}_{2} \mathrm{CO}$, or vice versa.

\section{Formation and Destruction of $\mathrm{OH}$ in the Interstellar Medium}

We summarize briefly the current ideas on the formation of interstellar molecules in general, with special attention to $\mathrm{OH}$. Three principle methods of formation have been considered. (a) By direct buildup in the gas by 2-body radiative recombination. (b) On particle surfaces which act as catalysts to remove excess energy. (c) From large

polyatomic molecules ejected from cool stars, either by providing solid surfaces for reactions with interstellar atoms, or by subsequent breakdown into the simpler molecules actually observed.

Optical data provide a less ambiguous determination of molecular abundances than do radio data, because there are no anomalous excitations of the optical transitions. Optical data for the star $\zeta$ Oph (Herbig, 1970) show no OH or $\mathrm{NH}$, and the ratios of these species to $\mathrm{CH}$ and $\mathrm{CH}^{+}$is well below the ratio which is predicted by formation of molecules on grains by chemical exchange (Stecher and Williams, 1966). On the other hand, radio observations of $\mathrm{CO}$ in other sources suggest an abundance well in excess of that predicted by chemical exchange.

Conversely, the predicted abundance ratios of several diatomic molecules according 
to the theory of formation by 2-body gaseous reactions do appear to fit the optical data for $\zeta$ Oph (Herbig, 1970). It has long been thought that the abundances produced by such mechanisms would be far less than the observed abundances. However the original calculation by Bates and Spitzer (1951) assumed typical interstellar H I cloud densities of no more than 20 atoms $\mathrm{cm}^{-3}$. Present data on interstellar lines (Herbig, 1968) shows however that densities required by the Bates-Spitzer theory are found in the dense $\mathrm{HI}_{\mathrm{I}}$ layer in front of $\zeta \mathrm{Oph}$, a cloud which also happens to be very abundant in molecules. Recent radio data of the continuum and recombination line emissions from Hil regions indicate typical densities of $\sim 500 \mathrm{~cm}^{-3}$ and as high as $5000 \mathrm{~cm}^{-3}$ in some cases. These values may be taken as representative of the densities that typify a sizable fraction of interstellar clouds. Furthermore, Solomon and Klemperer (1970) have revised the reaction rates for formation of molecules by radiative association, by including the effects of tunneling and trapping in the potential well. The revised rate coefficients of $3 \times 10^{-17}\left(\mathrm{CH}\right.$ formation) and $2 \times 10^{-16}\left(\mathrm{CH}^{+}\right.$formation) are a factor of 10 and 100 respectively greater than given by Bates and Spitzer. Formation by direct radiative association is faster than any possible formation on grain surfaces. Exothermic atom-molecule and ion-molecule reactions of $\mathrm{C}, \mathrm{C}^{+}, \mathrm{N}, \mathrm{O}$, and $\mathrm{H}$ with $\mathrm{CH}$ and $\mathrm{CH}^{+}$lead to the formation of other diatomic molecules including $\mathrm{H}_{2}, \mathrm{CN}$, and $\mathrm{CO}$ but not $\mathrm{NH}$ and $\mathrm{OH}$. The relative abundances of $\mathrm{CH}, \mathrm{CH}^{+}, \mathrm{CN}$, and $\mathrm{CO}$ are determined as a function of the density, temperature, radiation field, and age of an interstellar cloud. Solomon and Klemperer find excellent agreement with the observational data for $\zeta$ Oph, the Pleiades, and other regions.

Aside from the objection that molecule formation by exchange reactions on grains does not appear to produce the observed relative abundances, this process also requires the activation energy (typically $0.5 \mathrm{eV}$ ) to be provided by the kinetic energy of the incident atom. Molecule formation by this means is thus severely handicapped in $\mathrm{HI}$ regions where the kinetic energy is only about $10^{-3} \mathrm{eV}$. The energy can always be produced by special circumstances such as cloud-cloud collisions, or the driving of grains through the gas by radiation pressure from a hot star, but neither of these processes seems applicable to the case of $\zeta$ Oph or several other regions where molecular concentrations are found. In particular the large concentrations of $\mathrm{CO}$ in the $\mathrm{H}$ I regions surrounding the Orion Nebula cannot have formed from surface reactions on grains.

To produce $\mathrm{OH}$ without invoking grains we must invoke special reactions such as $\mathrm{O}+\mathrm{H}_{2} \rightleftarrows \mathrm{OH}+\mathrm{H}$, proposed by Carroll and Salpeter (1966). $\left(\mathrm{H}_{2}\right.$ has recently been discovered in large quantities $\left(\mathrm{H}_{2} / \mathrm{H}=0.3\right)$ in the direction of $\xi$ Persei (Carruthers, 1970).) For suitable rates, this reaction is assumed to occur at a temperature of $\sim 1000 \mathrm{~K}$, which is produced by cloud-cloud collisions. At such temperatures, the reaction rate exceeds that of any surface reactions, or of photodissociation. $\mathrm{OH} / \mathrm{H}$ ratios of $3 \times 10^{-4}$ to $10^{-8}$ can be produced, the variation depending directly on the abundance of $\mathrm{H}_{2}$. Where $\mathrm{H}_{2}$ is abundant, then so is $\mathrm{OH}$. This type of reaction is of particular relevance to the situation in which $\mathrm{OH}$ is detected in the vicinity of $\mathrm{HII}$ regions. It is very satisfactory that the abundance of $\mathrm{OH}$ found in these regions should 
be covered by the predictions, provided that the abundance of $\mathrm{H}_{2}$ is from $10 \%$ to equal the amount of atomic hydrogen in these regions (McNally, 1968).

In dust clouds there is another argument for the production of $\mathrm{OH}$ from the Carroll-Salpeter reaction. Heiles (1968) finds a definite lack of $21-\mathrm{cm}$ emission from several of these clouds (others have not been observed) and concludes that virtually all hydrogen must be in the form of $\mathrm{H}_{2}$. The strong absorption of background starlight by these clouds indicates the presence of much dust, and at the low temperatures found in these clouds $(<20 \mathrm{~K}), 2$-body radiative recombination would be inadequate for production of the $\mathrm{H}_{2}$. It is presumably formed by physical adsorption on the grains, a process which occurs only if the grain temperature is less than $\sim 7 \mathrm{~K}$. However, although $\mathrm{OH}$ in the clouds may be formed either by the exchange reaction [G] $\mathrm{H}+\mathrm{O} \rightarrow[\mathrm{G}]+\mathrm{OH}$ or by the Carroll-Salpeter reaction $\mathrm{H}_{2}+\mathrm{O} \rightarrow \mathrm{OH}+\mathrm{H}$, the latter dominates because the density of $\mathrm{H}_{2}$ is so very high. Even this reaction proceeds very slowly when temperatures are less than $20 \mathrm{~K}$, but may succeed in producing sufficient $\mathrm{OH}$ since $\mathrm{OH} / \mathrm{O}$ is only about $10^{-5}$ in the clouds. At such low temperatures the only process destroying $\mathrm{OH}$ would be photodissociation, on a time scale of $\sim 10^{8} \mathrm{yr}$.

Alternatively, it may be incorrect to picture the $\mathrm{OH}$ in dust clouds as forming under the present conditions. It may have instead been formed in the past, when temperatures were higher but when dust extinction was still large enough to cause considerable dimming of starlight. In this regard, there is some evidence based on multiple components in the observed $\mathrm{OH}$ features, that there is large-scale supersonic turbulence, with perhaps 2 or 3 main components, in some of the clouds. These components may have undergone collisions in the past, producing higher temperatures and enhanced molecular formation rates.

Although the Carroll-Salpeter reaction seems capable of producing the observed abundance of $\mathrm{OH}$ seen in absorption against continuum sources, and possibly also in dust clouds, it is not helpful in explaining the apparent association of $\mathrm{OH}$ and $\mathrm{H}_{2} \mathrm{O}$ emission sources, nor the evident correlation of $\mathrm{OH}$ and $\mathrm{H}_{2} \mathrm{CO}$ throughout the galactic plane. One mechanism proposed by Gwinn et al. (1968) produces $\mathrm{OH}$ from the collisional dissociation of $\mathrm{H}_{2} \mathrm{O}: \mathrm{H}+\mathrm{H}_{2} \mathrm{O}+4.5 \mathrm{eV} \rightarrow \mathrm{OH}^{*}+2 \mathrm{H}$ and at the same time produces the $\mathrm{OH}$ in anomalously excited states which seem capable of producing the Class I maser emission observed to go together with the $\mathrm{H}_{2} \mathrm{O}$ emission. While this mechanism is satisfactory in producing the large concentrations of excited $\mathrm{OH}$ that seem necessary in these sources $\left(\mathrm{OH} / \mathrm{H}\right.$ is typically $\left.10^{-4}\right)$, it leaves open the question of formation of $\mathrm{H}_{2} \mathrm{O}$ itself. Gwinn et al. consider the $\mathrm{H}_{2} \mathrm{O}$ to be sputtered off grains containing ice mantles. This hypothesis is attractive for producing the $\mathrm{H}_{2} \mathrm{CO}$ also while not producing many other molecules, as observed. We must invoke the Stecher-Williams chemical exchange reactions on grain surfaces. If the grain surfaces are ice, the only molecules produced are $\mathrm{H}_{2}$ and $\mathrm{CO}$ (aside from $\mathrm{H}_{2} \mathrm{O}$ which is sputtered off directly). The free $\mathrm{H}$ atoms performing the sputtering then may combine with the $\mathrm{CO}$ to produce $\mathrm{H}_{2} \mathrm{CO}$.

Such a picture seems consistent with the majority of molecular clouds which exhibit 
$\mathrm{OH}, \mathrm{H}_{2} \mathrm{CO}$, and in fewer cases, $\mathrm{H}_{2} \mathrm{O}$. However, we should not expect to find $\mathrm{CN}$ or $\mathrm{HCN}$ in these regions since the production of $\mathrm{CN}, \mathrm{NH}, \mathrm{N}_{2}$, as well as $\mathrm{OH}$, by surface exchange reaction can only occur if the grains are graphite. $\mathrm{H}_{2} \mathrm{O}$ would then have to form from the $\mathrm{OH}$, failing to produce the almost certain over-abundance of $\mathrm{H}_{2} \mathrm{O}$ relative to $\mathrm{OH}$. Unless several difierent molecular formation processes are proceeding at the same time, presumably in different parts of the cloud, it seems difficult to explain the four cases (Ori A, W51, W3 OH, DR $21 \mathrm{OH}$ ) in which $\mathrm{HCN}$ has recently been observed (Snyder and Buhl, 1970) in the same direction as maser emission from $\mathrm{H}_{2} \mathrm{O}$ and $\mathrm{OH}$. Many more observations are needed, however, especially for the most recently discovered molecules $\mathrm{CO}, \mathrm{CN}$, and $\mathrm{HCN}$, before the patterns that we must explain will fully emerge. As the most ubiquitous interstellar molecule so far discovered, $\mathrm{OH}$ can be expected to play a major role in determining the molecular processes that occur in dense interstellar clouds.

\section{References}

Altenhoff, W. J., Downes, D., Goad, L., Maxwell, A., and Rinehart, R.: 1970, Astron. Astrophys., Suppl. Series 1, 319.

Bates, D. R. and Spitzer, L., Jr.: 1951, Astrophys. J. 113, 441.

Carroll, T. O. and Salpeter, E. E.: 1966, Astrophys. J. 143, 609.

Carruthers, G. R.: 1970, Astrophys. J. Letters, in press.

Clark, B. G.: 1965, Astrophys. J. 142, 1398.

Davies, R. D.: 1969, private communication.

Goss, W. M.: 1968, Astrophys. J. Suppl. 15, 131.

Goss, W. M., Manchester, R. N., and Robinson, B. J.: 1970, Australian J. Phys., in press.

Gwinn, W. D., Millikan, R., Goss, W. M., and Turner, B. E.: 1968, unpublished.

Heiles, C. E.: 1968, Astrophys. J. 151, 919.

Heiles, C. E.: 1969, Astrophys. J. 157, 123.

Herbig, G. H.: 1968, Z. Astrophys. 68, 243.

Herbig, G. H.: 1970, Paper presented at 132nd Meeting of the American Astronomical Society, Boulder, Colo.

Litvak, M. M.: 1969, Astrophys. J. 156, 471.

Manchester, R. N. and Gordon, M. A.: 1970, private communication.

Manchester, R. N., Robinson, B. J., and Goss, W. M.: 1970, Australian J. Phys., in press.

McNally, D.: 1968, in Advances in Astronomy and Astrophysics, Vol. 6 (ed. by Z. Kopal) Academic Press, New York, London.

Mezger, P. G. and Henderson, A. P.: 1967, Astrophys. J. 147, 471.

Miley, G. K., Turner, B. E., Balick, B., and Heiles, C. E.: 1970, Astrophys. J. Letters 160, L119.

Reddish, V. C.: 1967, Monthly Notices Roy. Astron. Soc. 135, 251.

Robinson, B. J., Gardner, F. F., von Damme, K. J., and Bolton, J. G.: 1964, Nature 202, 989.

Robinson, B. J., Goss, W. M., and Manchester, R. N.: 1970, Australian J. Phys., 23, 363.

Sharpless, S.: 1959, Astrophys. J. Suppl. 4, 257.

Snyder, L. E. and Buhl, D.: 1970, in preparation.

Solomon, P. M. and Klemperer, W.: 1970, Paper presented at 132nd Meeting of the American Astronomical Society, Boulder, Colo.

Stecher, T. P. and Williams, D. A.: 1966, Astrophys. J. 146, 88.

Turner, B. E.: 1969a, Astrophys. J. 157, 103.

Turner, B. E.: 1969b, Astron. J. 74, 985.

Turner, B. E.: 1970, Astrophys. Letters (August issue).

Turner, B. E. and Heiles, C. E.: 1970, Paper presented at XIVth General Assembly, IAU, Brighton, England.

Turner, B. E., Balick, B., Cudaback, D. D., and Heiles, C. E.: 1970, in preparation. 
Webster, W. J. and Altenhoff, W. J.: 1970, Astrophys. Letters 5, 233.

Weinreb, S., Barrett, A. H., Meeks, M. L., and Henry, J. C.: 1963, Nature 200, 829.

Wilson, R. W., Jefferts, K. B., and Penzias, A. A.: 1970, Astrophys. J. Letters 161, L43.

Wilson, T. L.: 1970, in preparation.

Zuckerman, B. M., Buhl, D., Palmer, P., and Snyder, L. E.: 1970, Astrophys. J. 160, 485.

\section{DISCUSSION}

Turner: Limits for $\mathrm{OH}$ in small globules $\left(<3^{\prime}\right.$ in size) are not sufficient to indicate whether the $\mathrm{OH}$ abundance is less in these globules than in dust clouds. However, in larger globules, where the beam dilution is not so great, limits are adequate to state that the $\mathrm{OH}$ abundances are less than in those dust clouds.

Bok B. J.: Steward Observatory Reprint No. 26 contains a sample listing of positions (1975) and physical data for Unit Dark Nebulae, from large, single dark nebulae to large and small globules. Copies will be sent upon request. 